\title{
Low Dose and Time Efficient Molar Fraction STXM Analysis for Binary Material Systems
}

\author{
Markus Meyer ${ }^{1}, \underline{A n d r e a s ~ S p a ̈ t h}^{1}$ and Rainer H. Fink ${ }^{1, *}$ \\ 1. Physical Chemistry II, Friedrich-Alexander-Universität Erlangen-Nürnberg, Erlangen, Germany. \\ * Corresponding author, rainer.fink@fau.de
}

Composition and component analysis is a strong and well-known tool in STXM data post-treatment analysis to derive quantitative information on the micro-morphology of investigated specimens[1]. Different approaches have been published like Principal Component Analysis (PCA) [2] and Singular Value Decomposition (SVD) [3]. The present approach is related to SVD and likewise benefits from a multiple times higher time efficiency, which is accompanied by lower dose entry into the specimen. Such conditions offer the possibility to scan larger areas and hence promote higher sample statistics better representing the sample average.

The novel approach is an enhancement to the widely accepted standard procedure to exclude thickness or density related contrast. As described in detail elsewhere [4], the division of an image recorded at resonant photon energy and an image recorded with an photon energy well above the absorption edge delivers qualitative information on chemical (and/or dichroism related) contrast. This procedure, however, can be at the expense of image contrast (which is information depth), if the investigated material system consists out of compounds with strongly deviating carbon weight content per volume unit. Hence, depending on the material intrinsic carbon atom density the qualitative information is hampered.

The present study shows a mathematically proven alternative approach to overcome potential contrast blurring. It is demonstrated that the thickness levels out for two images (converted to optical density) recorded at any energy when divided by each other. Hence, a proper choice of photon energy which are resonant energies leading to contrast inversion in the qualitative images amplifies contrast enhancement. Beyond this, contrast inversion can be utilized as a sensitive measure for the quantitative composition, as each pixel volume contains a specific component ratio (= molar fraction ratio), which will return a unique, scalable ratio value upon division. Those ratio values can be simulated in dependence of molar fraction values from the pure spectra of the initial components, which are recorded independent from dose exposure to the sample of interest. The simulated values are then suitably fitted by a polynomial fit generating an inverse function of molar fraction values in dependence of the ratio values. In a final step the ratio values from each pixel are transformed into molar fraction values, quantitatively revealing the component distribution in the investigated area by resourcefully utilizing the qualitative information of only two images.

Limitation is found in the signal-to-noise ratio leading to an increase in statistic blurring, mainly governed by the detector setup. Beneficial side effects can furthermore be found in reduced positional drifts due to focus or energy positioning, which especially for low-contrast specimen can be hardly fixed by post-treatment procedures. The here presented theoretical consideration was successfully tested and applied in the framework of binary organic photovoltaic thin film systems with defined compound mixing values for the donor and acceptor material (cf. figure 1). Details on the investigated material systems and preparation can be found elsewhere [5]. The achieved results show that the presented 
approach complies very well with the expected values for the overall component distribution defined by the preparation mixture averaged over the sample. Furthermore, this analysis is completed by histograms of the calculated molar fraction values, proving reproducibility for different specimen areas thus allowing further statistical analysis. As shown in figure 2, the wider distribution around the expectancy value for higher processing temperature indicates a higher phase separation of the involved material components. The smaller the distribution in the histogram, the higher is the overall homogeneity within the sample.

This concept can be extended and applied to multinary systems with increasing statistical uncertainties. For serious results, the number of utilized qualitative image sets recorded at different energies has to be at least equal to the number of components which are to be determined [6].

\section{References:}

[1] IN Koprinarov et al, J. Phys. Chem. B 106 (2002) 5358.

[2] M Lerotic et al, J. Electron. Spectrosc. 144 (2005) 1137.

[3] X Zhang et al, J. Struc. Biol. 116 (1996) 335.

[4] J Stöhr, „NEXAFS Spectroscopy“, (Springer Verlag, Berlin, 1992).

[5] NS Güldal et al, J. Mater. Chem. A 4 (2016) 16136.

[6] The authors acknowledge funding from FAU Graduate School Molecular Science (GSMS), the German Research Foundation (DFG) within GRK1896 and the BMBF (contract 05 K16 WED).

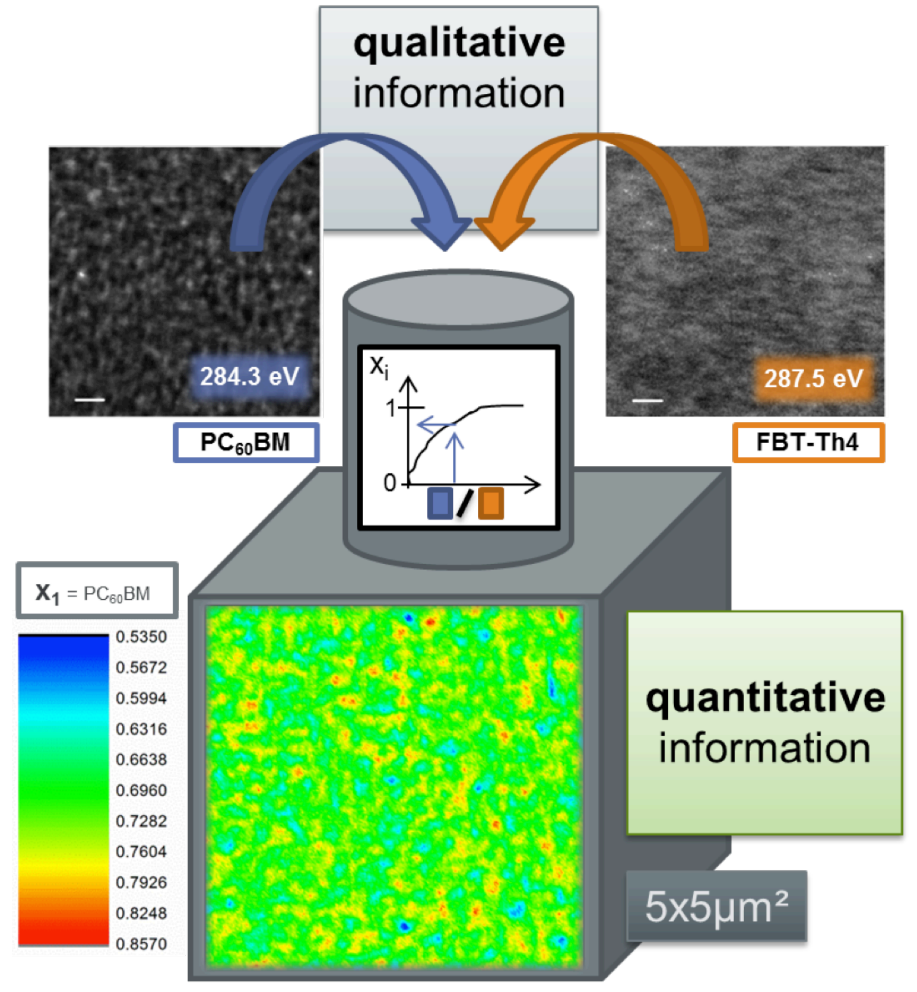

Figure. 1. Molar fraction map (colored) of binary material system, developed on the basis of only two microscopic images (and the pure component spectra) allowing intuitive quantitative evaluation and comparison of the data. This novel approach outstands due to time efficiency and marginal dose entry.
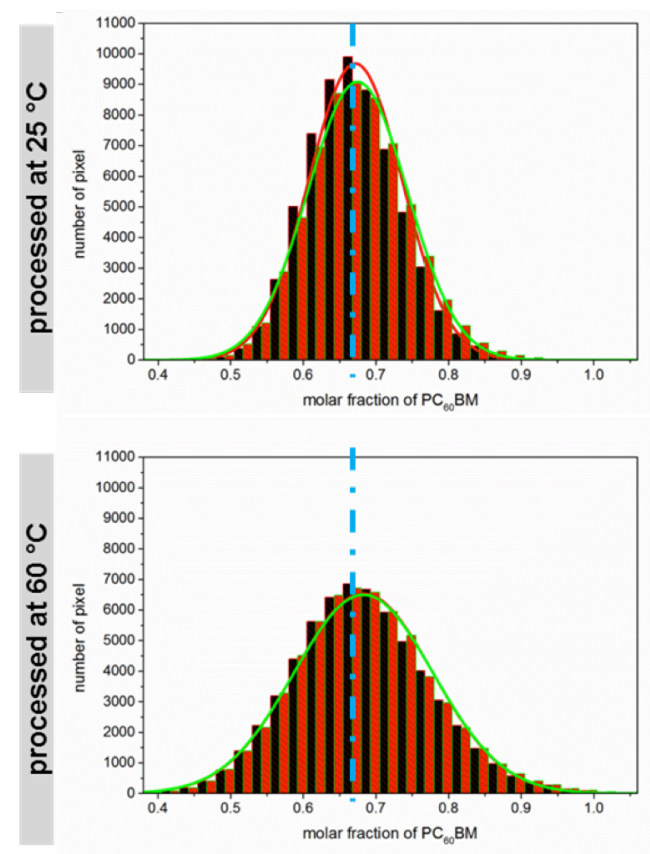

Figure. 2. Histograms of quantitative molar fraction images for two different sample areas (red/black) and two different preparation temperatures. The expectancy value from preparation mixture is marked by blue dotted line. Wider distributions of the molar fraction values indicate stronger phase separation. 